\title{
INTERSECTING UNIONS OF MAXIMAL CONVEX SETS
}

\author{
MARILYN BREEN
}

\begin{abstract}
Hare and Kenelly have characterized the intersection of the maximal starshaped subsets of a set $S$, where $S$ is compact, simply connected and planar, and Sparks has solved the general problem for maximal $L_{n}$ sets. In this paper, a similar question is examined for unions of maximal convex sets: Let $S$ be a subset of $R^{2}, \mathscr{C}$ the collection of all maximal convex subsets of $S$, and $\mathscr{N}=$ $\{A \cup B: A, B$ distinct members of $\mathscr{C}\}$. Then $\bigcap \mathscr{N}$ is expressible as a union of three or fewer convex sets.
\end{abstract}

\section{Intersecting unions of two maximal convex sets.}

Lemma 1. Let $\mathscr{C}$ be any family of sets and $\mathscr{M}=\left\{A_{1} \cup \cdots \cup A_{k}: A_{1}, \cdots\right.$, $A_{k}$ distinct members of $\left.\mathscr{C}\right\}$. Then $x \in \bigcap \mathscr{M}$ if and only if there are at most $k-1$ members of $\mathscr{C}$ which fail to contain $x$.

THEOREM 1. Let $\mathscr{C}$ be any collection of closed convex subsets of the plane and let $\mathscr{M}=\{A \cup B: A, B$ distinct members of $\mathscr{C}\}$. Then $\bigcap \mathscr{M}$ can be expressed as a union of three or fewer closed convex sets.

Proof. We assume that $\bigcap \mathscr{M}$ is not convex and consists of more than three points, and that $\mathscr{C}$ has at least three distinct members, for otherwise the result is trivial. We examine two cases.

Case 1. Assume that $\bigcap \mathscr{M}$ is three convex. That is, for $x, y, z$ in $\bigcap \mathscr{M}$, at least one of the corresponding segments lies in $\bigcap \mathscr{M}$. Since $\bigcap \mathscr{M}$ is closed, if it is connected, then by a theorem of Valentine [3], $\cap \mathscr{M}$ is expressible as a union of three or fewer closed convex sets having a nonempty intersection, completing the proof. If $\cap \mathscr{M}$ is not connected, then it has exactly two closed components, each of which is necessarily convex by the three convexity of $\bigcap \mathscr{M}$. This completes Case 1 .

Case 2. If $\bigcap \mathscr{M}$ is not three convex, there are points $x, y, z$ in $\bigcap \mathscr{M}$ for which none of the corresponding segments lie in $\bigcap \mathscr{M}$. Thus there is some $A \cup B$ in $\mathscr{M}$ not containing all three segments. Assume $x, y \in A$, $z \in B \sim A$. Then $A$ is the only member of $\mathscr{C}$ not containing $z$ (by Lemma 1 ). Since $[x, y] \ddagger \bigcap \mathscr{M}$, there is some $C \cup D$ in $\mathscr{M}$ not containing $[x, y]$, and without loss of generality we may assume $x \in C \sim D, y \in D \sim C, z \in D$.

Received by the editors September 5, 1972 and, in revised form, November 15, 1972. AMS (MOS) subject classifications (1970). Primary 52A10, 52A40.

Key words and phrases. Maximal convex subsets, unions of convex sets.

(c) American Mathematical Society 1973 
Again by Lemma $1, D$ is the only member of $\mathscr{C}$ not containing $x, C$ the only one not containing $y$. Also, since $y, z \in D,[y, z] \subseteq D$. Since $y \in$ $A \sim C, A \neq C$, and $A \cup C$ belongs to $\mathscr{M}$. Thus $z \in C$ and $[x, z] \subseteq C$.

Moreover, for any member $E$ of $\mathscr{C}$ distinct from each of $A, C, D$, by Lemma $1, E$ necessarily contains $x, y$, and $z$.

We examine the following closed convex subsets of $\cap \mathscr{M}$. Define $A_{0}=\bigcap\{E: E$ in $\mathscr{C}, E \neq A\}, C_{0}=\bigcap\{E: E$ in $\mathscr{C}, E \neq C\}, D_{0}=\bigcap\{E: E$ in $\mathscr{C}$, $E \neq D\}$. We will show that the only points of $\cap \mathscr{M}$ which fail to be in $A_{0} \cup C_{0} \cup D_{0}$ necessarily lie in $A \cap C \cap D \subseteq \operatorname{ker}(\cap \mathscr{M})$. It will then be easy to express $\cap \mathscr{M}$ as a union of three closed convex sets.

We begin by showing that $A \cap C \cap D$ is in $\operatorname{conv}\{x, y, z\}$. Let $p \in$ $A \cap C \cap D$. If $[y, z]$ contained $p$, then $[y, p] \subseteq A,[p, z] \subseteq C$, and $[y, z] \subseteq$ $A \cup C$. However $[y, z] \subseteq E$ for $E \neq A, C, D$. Also $[y, z] \subseteq D$, so $[y, z]$ would lie in $\cap \mathscr{M}$, a contradiction since none of the segments determined by $x, y, z$ lie in $\bigcap \mathscr{M}$.

A parallel argument shows that neither $[x, y]$ nor $[x, z]$ contains a point of $A \cap C \cap D$. Now for $p$ in $A \cap C \cap D$, if $[p, x]$ cut $[y, z]$ at $q$, then $[p, x] \subseteq A \cap C$ and $q \in A \cap C \cap D$ which is impossible by the preceding paragraph. Similarly $[p, y]$ cannot cut $[x, z],[p, z]$ cannot cut $[x, y]$.

Also, $x \notin \operatorname{conv}\{p, y, z\}$ (for otherwise $x$ would lie in $D$ ), $y \notin \operatorname{conv}\{p, x, z\}$, and $z \notin \operatorname{conv}\{p, x, y\}$.

Hence $p$ must be interior to $\operatorname{conv}\{x, y, z\}$, and since $x, y, z \in E$ for every $E \neq A, C, D$, it follows that $p$ is in every member of $\mathscr{C}$ and in $\bigcap \mathscr{M}$.

Moreover, $p \in \operatorname{ker}(\bigcap \mathscr{M})$, for if $t \in \bigcap \mathscr{M}, t$ fails to belong to at most one $E$ in $\mathscr{C}$, so $[p, t]$ fails to lie in at most one member of $\mathscr{C}$, and $[p, t] \subseteq$ $\cap \mathscr{M}$.

Now examine the sets $A_{0}, C_{0}, D_{0}$ defined previously. It is clear that each of these sets lies in $\bigcap \mathscr{M}$ by Lemma 1. For $u$ in $\bigcap \mathscr{M}$, either $u$ fails to lie in one of $A, C$, or $D$ (and hence lies in one of $A_{0}, C_{0}$, or $D_{0}$ ), or $u$ lies in $A \cap C \cap D$. Since $A \cap C \cap D \subseteq \operatorname{ker}(\cap \mathscr{M})$, the set

$$
\operatorname{conv}\left((A \cap C \cap D) \cup A_{0}\right)
$$

is a subset of $\cap \mathscr{M}$. Thus each of the sets $A_{1}=\operatorname{cl} \operatorname{conv}\left((A \cap C \cap D) \cup A_{0}\right)$, $C_{1}=\mathrm{cl} \operatorname{conv}\left((A \cap C \cap D) \cup C_{0}\right), D_{1}=\mathrm{cl} \operatorname{conv}\left((A \cap C \cap D) \cup D_{0}\right)$ is a closed convex subset of the closed set $\cap \mathscr{M}$, and $\cap \mathscr{M}=A_{1} \cup C_{1} \cup D_{1}$, completing the proof.

REMARK. It is easy to find examples which show that the number three in Theorem 1 is best possible. (See Example 1 of this paper.)

Using Theorem 1, it is possible to prove the following.

THEOREM 2. Let $S$ be planar, $\mathscr{C}$ the collection of all maximal convex subsets of $S$. Let $\mathscr{N}=\{A \cup B: A, B$ distinct members of $\mathscr{C}\}$. Then $\cap \mathscr{N}$ can be expressed as a union of three or fewer convex sets. 
Proof. By an easy application of Theorem 1, for $\mathscr{M}=\{\operatorname{cl} A \cup \mathrm{cl} B$ : $A, B$ distinct members of $\mathscr{C}\}, \cap \mathscr{M}$ is a union of three or fewer closed convex sets $S_{i}, i=1,2,3$.

Let $M_{i}=S_{i} \cap(\cap \mathscr{N}), i=1,2,3$. If each $M_{i}$ is convex, the proof is complete. Assume otherwise to reach a contradiction. Suppose for $v, w$ in $M_{1},[v, w] \nsubseteq M_{1}$. Then for some $p, v<p<w, p \notin M_{1}$. Therefore, there exist sets $G, F$ in $\mathscr{C}$ with $p \notin G \cup F$. Without loss of generality, assume $v \in G \sim F, w \in F \sim G$.

If $G, F$ are the only members of $\mathscr{C}$, the proof is trivial. Otherwise, for every $E$ in $\mathscr{C} \sim\{G, F\},[v, w] \subseteq E$ by Lemma 1 . Thus $p \in[v, w] \subseteq E \subseteq S$. Also, since $p \in S_{1}, p \in \operatorname{cl} G \cup \operatorname{cl} F$, so assume $p \in \operatorname{cl} G$. For every $x$ in $G$, if $[p, x] \subseteq S$, then the cone $p G \equiv \bigcup\{[p, x]: x$ in $G\}$ would be a convex subset of $S$ containing $G$. But since $G$ is maximal, this would imply that $p \in G$, a contradiction. Hence for some $x$ in $G,[x, p] \nsubseteq S$. Clearly such an $x$ cannot lie on the line $L(v, w)$ determined by $v$ and $w$, since $[v, w] \subseteq S$ and $[x, v] \subseteq G \subseteq S$.

For some $y, x<y<p, y \notin S$. Since $x, p \in \operatorname{cl} G, y \in \operatorname{cl} G$, and since $G$ is convex, $y$ must lie on bdry $G$. There is a supporting hyperplane $H$ to $\mathrm{cl} G$ at $y$, and $H$ contains $[x, p]$ since $[y, p] \subseteq \operatorname{cl} G \sim G \subseteq$ bdry $G$. Note that this implies $x \in$ bdry $G$, and therefore $p$ sees via $S$ all points interior to $G$. Clearly int $G \neq \varnothing$ since $x \notin L(v, w)$.

Consider the cone $G_{1}=p($ int $G) \equiv \bigcup\{[p, x]: x \in$ int $G\}$. This is a convex subset of $S$. If necessary, extend $G_{1}$ to a maximal convex subset $G_{2}$ of $S$. It is easy to see that $w \notin G_{2}$ : Let $U$ be any spherical neighborhood of $x$ disjoint from the line $L(w, y)$. Certainly $U$ contains points of int $G$, and for $x_{1}$ in $U \cap$ int $G, y \in \operatorname{conv}\left\{x_{1}, p, w\right\}$. If $w$ were in $G_{2}$, then $y \in G_{2} \subseteq S$, a contradiction since $y \notin S$.

Now $p \in G_{2} \sim G$, so $G \neq G_{2}$ and $G \cup G_{2}$ is in $\mathscr{N}$. Since $w \in M_{1} \subseteq \bigcap \mathscr{N}$, $w$ must lie in $G \cup G_{2}$, but this is clearly impossible by the preceding paragraph. Hence our assumption is false, each $M_{i}$ is convex, and $\cap \mathscr{N}$ is a union of three or fewer convex sets.

2. The general case. It would be interesting to obtain analogues of Theorems 1 and 2 for unions of $k$ convex sets. The following results, although for special cases, invite the conjecture that the appropriate bound is $k(k+1) / 2$.

THEOREM 3. Let $\mathscr{C}$ be any collection of $k+1$ closed convex subsets of the plane and let $\mathscr{M}=\left\{A_{1} \cup \cdots \cup A_{k}: A_{1}, \cdots, A_{k}\right.$ distinct members of $\left.\mathscr{C}\right\}$. Then $\cap \mathscr{M}$ is expressible as a union of $k(k+1) / 2$ or fewer closed convex sets. The result is best possible for all $k$.

Proof. The proof is by induction. The result is trivial for $k=1$, and for $k=2$, the result is an immediate consequence of Theorem 1. Assume the theorem true for $2<k-1$ to prove for arbitrary $k$. 
Select any set $A$ in $\mathscr{C}$ and define subsets $P, Q$ of $\cap \mathscr{M}$ in the following manner. Let

$P=\{x: x \in A$ and $x$ fails to lie in exactly $k-1$ members of $\mathscr{C} \sim\{A\}\}$,

$Q=\{x: x$ fails to lie in at most $k-2$ members of $\mathscr{C} \sim\{A\}\}$.

Note that $x \in Q$ if and only if either $x \in A$ and $x$ fails to lie in no more than $k-2$ members of $\mathscr{C}$ or $x \notin A$ and $x$ fails to lie in no more than $k-1$ members of $\mathscr{C}$. Using Lemma 1 , it is clear that $P \cup Q=\bigcap \mathscr{M}$.

Examine the set $Q$. Now $\mathscr{C} \sim\{A\}$ is a collection of $k$ closed convex sets in the plane. Letting

$$
\mathscr{N}=\left\{B_{1} \cup \cdots \cup B_{k-1}: B_{1}, \cdots, B_{k-1} \text { distinct members of } \mathscr{C} \sim\{A\}\right\},
$$

by our induction hypothesis, $Q=\bigcap \mathscr{N}$ is expressible as a union of $(k-1) k / 2$ or fewer closed convex sets.

Furthermore, any point of $P$ necessarily lies in exactly two members of $\mathscr{C}$, one of which is $A$. Letting $E_{i}=A \cap A_{i}, A_{i}$ in $\mathscr{C} \sim\{A\}, 1 \leqq i \leqq k$, then $P=\bigcup_{i=1}^{k} E_{i}$.

Hence $P \cup Q=\bigcap \mathscr{M}$ is a union of $(k-1) k / 2+k=k(k+1) / 2$ or fewer closed convex sets, completing the proof.

Example 1. To see that the result in Theorem 3 is best possible, let $\mathscr{C}$ denote a collection of $k+1$ lines $L_{i}, 1 \leqq i \leqq k+1$, every two intersecting and no three having a common point. Then the corresponding $\cap \mathscr{M}$ consists of exactly $k(k+1) / 2$ isolated points.

In conclusion, we note that Example 1 reveals the "worst" case when $\mathscr{C}$ is any family of lines, for a proof paralleling that of Theorem 3 shows that the bound is again $k(k+1) / 2$. The only additional step involves showing that for $A$ in $\mathscr{C}$, the corresponding $P$ may be represented as a union of $k$ or fewer convex sets: If more than $k$ convex sets were required, there would be at least $k+1$ distinct members of $\mathscr{C} \sim\{A\}$, each intersecting $A$ at a different point, and for $x$ in $A \cap(\cap \mathscr{M}), x$ would fail to lie in at least $k$ members of $\mathscr{C}$, contradicting Lemma 1 . Thus $P$ has the desired representation and the result follows.

\section{REFERENCES}

1. W. R. Hare and J. W. Kenelly, Intersections of maximal starshaped sets, Proc. Amer. Math. Soc. 19 (1968), 1299-1302. MR 38 \#1605.

2. A. G. Sparks, Intersections of maximal $L_{n}$ sets, Proc. Amer. Math. Soc. 24 (1970), 245-250. MR 40 \#6368.

3. F. A. Valentine, A three point convexity property, Pacific J. Math. 7 (1957), 1227-1235. MR 20 \#6071.

Department of Mathematics, University of Oklahoma, Norman, Oklahoma 73069 